\title{
ON COEFFICIENT DOMINATION FOR SOME CLASSES OF ANALYTIC FUNCTIONS
}

\author{
S. Perera and D.R. Wilken \\ Union College, Department of Mathematics, Bailey Hall \\ Schenectady, NY 12308-2311, U.S.A. \\ University at Albany, State University of New York, Department of Mathematics and Statistics \\ Earth Science 110, Albany, NY 12222, U.S.A.
}

Abstract. For $\alpha \leq 1$ and $\beta \geq 0$, let $\mathscr{A}_{\alpha, \beta}$ denote the set of functions $f$ analytic in $\mathbf{D}=\{z \in \mathbf{C}:|z|<1\}$ such that $f(0)=0, f^{\prime}(0)=1$ and

$$
\left|\arg \frac{z f^{\prime}(z)}{e^{i \gamma} s(z)}\right| \leq \beta \cdot \frac{\pi}{2} \quad \text { for } z \quad \text { in } \mathbf{D}
$$

for some $s$ in $S t(\alpha)$, the starlike functions of order $\alpha(S t(1)=\{z\})$ and some real number $\gamma$. For $q>0$ and $|p| \leq q$, let $\mathscr{V}_{p, q}$ denote the usual subfamilies of functions having bounded boundary rotation. It is shown that a coefficient domination for $\mathscr{V}_{p, q}^{\prime}$, in the range $p \geq 0$ and $q \geq \max \{p, 2\}$, extends to $\mathscr{A}_{\alpha, \beta}^{\prime}$ in a corresponding range for $\alpha$ and $\beta$. (The family $\mathscr{F}^{\prime}$ denotes the set of derivatives of the functions in the family $\mathscr{F}$.) Examples are constructed to show that the range of parameters in both cases is sharp for the second coefficient. As a consequence we obtain following the proper inclusions: If $\varepsilon=2(1-\alpha)$ and $\beta+\frac{1}{2} \varepsilon<1$, then

$$
\overline{\operatorname{co}} \mathscr{A}_{\alpha, \beta}^{\prime} \supsetneqq \overline{\operatorname{co}} \mathscr{V}_{\varepsilon, 2 \beta+\varepsilon}^{\prime} \supsetneqq \mathscr{F}_{\beta, \beta+\varepsilon}
$$

where, for positive numbers $a$ and $b$ and with $U=\{x \in \mathbf{C}:|x|=1\}$,

$$
\mathscr{F}_{a, b}=\left\{\int_{U \times U} \frac{(1-x z)^{a}}{(1-y z)^{b}} d \mu(x, y): \mu \text { is a probability measure on } U \times U\right\} .
$$

We also extend and organize a variety of results in the literature for the families $\mathscr{A}_{\alpha, \beta}, \mathscr{V}_{p, q}$ and $\mathscr{F}_{a, b}$ and identify the form of the support points of $\mathscr{A}_{\alpha, \beta}$ and $\mathscr{V}_{p, q}$.

1991 Mathematics Subject Classification: Primary 30C45; Secondary 30C50. 


\section{Introduction}

Let $\mathscr{A}$ denote the set of all functions analytic in $\mathbf{D}=\{z \in \mathbf{C}:|z|<1\}$. Then $\mathscr{A}$ is a locally convex linear topological space under the topology of uniform convergence on compact subsets of $\mathbf{D}$.

For $\alpha \leq 1$ and $\beta \geq 0$, let $\mathscr{A}_{\alpha, \beta}$ denote the set of functions $f$ in $\mathscr{A}$ such that $f(0)=0, f^{\prime}(0)=1$ and

$$
\left|\arg \frac{z f^{\prime}(z)}{e^{i \gamma} s(z)}\right| \leq \beta \cdot \frac{\pi}{2} \quad \text { for } \quad z \in \mathbf{D}
$$

for some $s$ in $S t(\alpha)$, the starlike functions of order $\alpha(S t(1)=\{z\})$ and for some real number $\gamma$. These families were introduced in [15]. Note that $f \in \mathscr{A}_{\alpha, 0}$ if and only if $f(0)=0, f^{\prime}(0)=1$ and $z f^{\prime}(z) \in S t(\alpha)$. Alternatively, $\mathscr{A}_{\alpha, 0}=\mathscr{K}(\alpha)$, the family of convex functions of order $\alpha$. Note also that $\mathscr{A}_{0, \beta}=\mathscr{C}(\beta)$, the family of close-to-convex functions of order $\beta$ introduced in [16]. (See also [10].)

For $q>0$ and $|p| \leq q$, let $\mathscr{V}_{p, q}$ denote the set of functions $f$ in $\mathscr{A}$ such that $f(0)=0, f^{\prime}(0)=1$ and

$$
f^{\prime}(z)=\exp \left\{-\int_{U} \log (1-x z) d \mu(x)\right\}
$$

where $U=\{z \in \mathbf{C}:|z|=1\}$ and $\mu$ is a real measure on $U$ satisfying $\int_{U} d \mu=p$ and $\int_{U}|d \mu| \leq q$. These families were introduced in [3]. $\mathscr{V}_{2, k}$ is the same as $\mathscr{V}_{k}$, the family of functions having boundary rotation at most $k \pi$. Also, if $p=q$ then $\mu=p \cdot \lambda$ where $\lambda$ is a probability measure on $U$. Hence $f \in \mathscr{V}_{q, q}$ if and only if $f(0)=0, f^{\prime}(0)=1$ and $z f^{\prime}(z) \in S t(\alpha)$, where $q=2(1-\alpha)$. We thus have $\mathscr{V}_{q, q}=\mathscr{A}_{\alpha, 0}$, where $q=2(1-\alpha)$.

For $a>0$ and $b>0$, let

$$
\mathscr{F}_{a, b}=\left\{\int_{U \times U} \frac{(1-x z)^{a}}{(1-y z)^{b}} d \mu(x, y): \mu \text { is a probability measure on } U \times U\right\} .
$$

Let $\mathscr{F}$ be a compact subset of $\mathscr{A}$. A function $f$ in $\mathscr{F}$ is a support point of $\mathscr{F}$ if there is a continuous linear functional $J$ on $\mathscr{A}$ such that

$$
\operatorname{Re} J(f)=\max \{\operatorname{Re} J(g): g \in \mathscr{F}\}
$$

and $\operatorname{Re} J$ is nonconstant on $\mathscr{F}$. If the function $f$ uniquely maximizes $\operatorname{Re} J$ over $\mathscr{F}$, then $f$ is called an exposed point of $\mathscr{F}$. The set of support points of $\mathscr{F}$ is denoted by $\Sigma \mathscr{F}$, the closed convex hull of $\mathscr{F}$ is denoted by $\overline{c o} \mathscr{F}$ and the set of extreme points of $\overline{\mathrm{co}} \mathscr{F}$ is denoted by $\mathscr{E} \overline{\mathrm{co}} \mathscr{F}$. We use $\mathscr{F}^{\prime}$ to denote the set of derivatives of functions in $\mathscr{F}$, i.e. $\mathscr{F}^{\prime}=\left\{f^{\prime}: f \in \mathscr{F}\right\}$. 
In many places in the literature ([3], [5], [1], [10], [13], [15]) properties of the families $\mathscr{A}_{\alpha, \beta}, \mathscr{V}_{p, q}$ and $\mathscr{F}_{a, b}$ have been studied. Questions concerning their closed convex hulls, extreme points, support points, as well as inclusion relations, have been addressed. For example, using our terminology, in [5] it was shown that, for $k \geq 4$,

$$
\overline{\mathrm{co}} \mathscr{V}_{2, k}^{\prime}=\overline{\mathrm{co}} \mathscr{A}_{0, \frac{1}{2} k-1}^{\prime}=\mathscr{F}_{\frac{1}{2} k-1, \frac{1}{2} k+1} .
$$

In [1], [11], [14] and [15] it was shown that, for $\alpha<1$,

$$
\Sigma \mathscr{A}_{\alpha, 1}^{\prime}=\mathscr{E} \mathscr{F}_{1,3-2 \alpha}=\left\{\frac{1-x z}{(1-y z)^{3-2 \alpha}}:|x|=|y|=1, x \neq y\right\} .
$$

Additional results exist throughout the literature.

In this paper our goal is twofold. The first goal is to gather, extend and organize somewhat these disparate results and identify the form of support points of the families $\mathscr{A}_{\alpha, \beta}$ and $\mathscr{V}_{p, q}$. The second goal is to show that a coefficient domination for $\mathscr{V}_{p, q}^{\prime}$, in the range $p \geq 0$ and $q \geq \max \{p, 2\}$, extends to $\mathscr{A}_{\alpha, \beta}^{\prime}$ in a corresponding range for $\alpha$ and $\beta$, and to construct examples which show that the range of parameters in both cases is sharp for the second coefficient. As a consequence we obtain the following proper inclusions:

If $\varepsilon=2(1-\alpha)$ and $\beta+\frac{1}{2} \varepsilon<1$, then

$$
\overline{\operatorname{co}} \mathscr{A}_{\alpha, \beta}^{\prime} \supsetneqq \overline{\operatorname{co}} \mathscr{V}_{\varepsilon, 2 \beta+\varepsilon}^{\prime} \supsetneqq \mathscr{F}_{\beta, \beta+\varepsilon}
$$

\section{Convex hulls and extreme points}

Recall that a function $f$ in $\mathscr{A}$ is subordinate to a function $g$ in $\mathscr{A}$, denoted $f \prec g$, if there is a function $\varphi$ in $\mathscr{A}$ with $\varphi(0)=0,|\varphi(z)|<1$ for $z$ in $\mathbf{D}$, and such that $f(z)=g(\varphi(z))$ for $z$ in $\mathbf{D}$. The following reformulation of $\mathscr{A}_{\alpha, \beta}$ follows easily from the definition.

Lemma 1. A function $f$ in $\mathscr{A}$ with $f(0)=0$ belongs to $\mathscr{A}_{\alpha, \beta}$ if and only if $f^{\prime}(z)=p(z) s(z) / z$, where $p(z) \prec((1+c z) /(1-z))^{\beta}$, for some $|c|=1$, and $s$ belongs to $S t(\alpha)$.

For two families of functions $\mathscr{F}$ and $\mathscr{G}$ in $\mathscr{A}$, let

$$
\mathscr{F} \cdot \mathscr{G}=\{f \cdot g: f \in \mathscr{F}, g \in \mathscr{G}\} .
$$

We need the following useful lemma.

Lemma 2. Let $\mathscr{F}$ and $\mathscr{G}$ be compact subsets of $\mathscr{A}$. Then

i) $\mathscr{F} \cdot \mathscr{G}$ is compact.

ii) If $h \in \mathscr{E} \overline{\mathrm{co}}(\mathscr{F} \cdot \mathscr{G})$ and $h$ is not identically zero, then $h=f \cdot g$ where $f \in \mathscr{E} \overline{\mathrm{co}} \mathscr{F}$ and $g \in \mathscr{E} \overline{\mathrm{co}} \mathscr{G}$. 
Proof. i) The fact that $\mathscr{F} \cdot \mathscr{G}$ is compact follows easily from the properties of compact subsets of $\mathscr{A}$.

To establish ii $)$, let $h \in \mathscr{E} \overline{\mathrm{co}}(\mathscr{F} \cdot \mathscr{G})$. Then $h=f \cdot g$ where $f \in \mathscr{F}$ and $g \in \mathscr{G}$. If $f=t f_{1}+(1-t) f_{2}, 0<t<1, f_{1} \neq f_{2}, f_{1}, f_{2} \in \overline{c o} \mathscr{F}$, then $h=t f_{1} \cdot g+(1-t) f_{2} \cdot g$ where $f_{1} g, f_{2} g \in(\overline{\mathrm{co}} \mathscr{F}) \cdot \mathscr{G} \subseteq \overline{\mathrm{co}}(\mathscr{F} \cdot \mathscr{G})$. Since $h$ is an extreme point we must have $f_{1} g=f_{2} g$. But $f_{2} \neq f_{1}$ so $g \equiv 0$. Hence $h \equiv 0$, a contradiction. Thus $f \in \mathscr{E} \overline{\mathrm{co}} \mathscr{F}$. Similarly $g \in \mathscr{E} \overline{\mathrm{co}} \mathscr{G}$, as desired.

For $a>0, b>0$ let $k(z ; x, y)$ denote the function in $\mathscr{A}$ such that $k(0)=0$ and $k^{\prime}(z ; x, y)=(1-x z)^{a} /(1-y z)^{b},|x|=|y|=1$. Note that for each $a$ and $b$ we obtain a family of functions, although for simplicity of notation we suppress the $a$ and $b$. It will usually be clear in the context of any argument what family, i.e. what $a$ and $b$, is being discussed. The following well-known result ([3], [1]) will also be useful.

Lemma 3. Let $a>0, b>0$.

i) $\mathscr{E} \mathscr{F}_{a, b} \subseteq\left\{k^{\prime}(z ; x, y):|x|=|y|=1\right\}$.

ii) If $0<a \leq 1,\left\{k^{\prime}(z ; x, y):|x|=|y|=1, x \neq y\right\} \subseteq \mathscr{E}_{a, b}$.

iii) If $0<a \leq b$, then $k^{\prime}(z ; x, y) \notin \mathscr{E} \mathscr{F}_{a, b}$ when $x=y$.

iv) If $0<a \leq 1$ and $a \leq b$, then $\mathscr{E} \mathscr{F}_{a, b}=\left\{k^{\prime}(z ; x, y):|x|=|y|=1, x \neq y\right\}$.

Throughout this paper let $\varepsilon$ denote $2(1-\alpha)$. It is easy to verify that the functions $k(z ; x, y)$ such that $k(0)=0$ and

$$
k^{\prime}(z ; x, y)=\frac{(1-x z)^{\beta}}{(1-y z)^{\beta+\varepsilon}}, \quad \text { where }|x|=|y|=1,
$$

are (important) examples of functions in $\mathscr{A}_{\alpha, \beta}$.

Theorem 4.

i) Let $\beta \geq 1$. Then

$\overline{\operatorname{co}} \mathscr{A}_{\alpha, \beta}=\left\{\int_{U \times U} k(z ; x, y) d \mu(x, y): \mu\right.$ is a probability measure on $\left.U \times U\right\}$.

Equivalently $\overline{\operatorname{co}} \mathscr{A}_{\alpha, \beta}^{\prime \prime}=\mathscr{F}_{\beta, \beta+\varepsilon}$.

ii) Let $\beta>1$. Then

$$
\mathscr{E} \overline{\operatorname{co}} \mathscr{A}_{\alpha, \beta} \subseteq\{k(z ; x, y):|x|=|y|=1, x \neq y\} .
$$

iii) Let $\beta=1$. Then

$$
\mathscr{E} \overline{\operatorname{co}} \mathscr{A}_{\alpha, 1}=\{k(z ; x, y):|x|=|y|=1, x \neq y\} .
$$

iv) Let $0<\beta<1$. If $f \in \mathscr{E} \overline{\mathrm{co}} \mathscr{A}_{\alpha, \beta}$, then $f(0)=0$ and

$$
f^{\prime}(z)=\left(\frac{1+c \varphi(z)}{1-\varphi(z)}\right)^{\beta} \frac{1}{(1-y z)^{\varepsilon}},
$$

where $|y|=|c|=1, c \neq-1$ and $\varphi$ is an inner function with $\varphi(0)=0$. 
Proof. i) Using standard methods we show $\overline{\operatorname{co}} \mathscr{A}_{\alpha, \beta}^{\prime}=\mathscr{F}_{\beta, \beta+\varepsilon}$ as follows. If $f \in \mathscr{A}_{\alpha, \beta}$, by Lemma 1 we have that $f^{\prime}(z)=p(z) s(z) / z$. But $\beta \geq 1$ and $s \in S t(\alpha)$ imply that

$$
\begin{aligned}
& p(z)=\int\left(\frac{1+c u z}{1-u z}\right)^{\beta} d \lambda(u) \text { and } \\
& s(z)=\int \frac{z}{(1-v z)^{\varepsilon}} d \nu(v), \text { where } \lambda \text { and } \nu \text { are probability measures on } U .
\end{aligned}
$$

It follows that

$$
p(z) \frac{s(z)}{z}=\int \frac{(1-x z)^{\beta}}{(1-y z)^{\beta+\varepsilon}} d \mu(x, y),
$$

where $\mu$ is a probability measure on $U \times U$. Since $\mathscr{F}_{\beta, \beta+\varepsilon}$ is compact and convex and each $k^{\prime}(z ; x, y)=(1-x z)^{\beta} /(1-y z)^{\beta+\varepsilon} \in \mathscr{A}_{\alpha, \beta}^{\prime}$, we have $\overline{\operatorname{co}} \mathscr{A}_{\alpha, \beta}^{\prime}=\mathscr{F}_{\beta, \beta+\varepsilon}$.

ii) and iii) Apply Lemma 3 , respectively, to the cases $\beta>1$ and $\beta=1$.

iv) Let $f \in \mathscr{E} \overline{\mathrm{co}} \mathscr{A}_{\alpha, \beta}$. Again by Lemma $1, f^{\prime}(z)=p(z) \cdot s(z) / z$, where $p(z) \prec((1+c z) /(1-z))^{\beta}$ for some $|c|=1$ and where $s$ belongs to $\operatorname{St}(\alpha)$. Let

$$
\mathscr{P}_{c}=\left\{f \in \mathscr{A}: f \prec\left(\frac{1+c z}{1-z}\right)^{\beta}\right\} .
$$

In [1] it was shown that, for $0<\beta<1$ and $c=1$,

$$
\mathscr{E} \mathscr{P}_{1}=\left\{\left(\frac{1+\varphi}{1-\varphi}\right)^{\beta}: \varphi \text { is an inner function with } \varphi(0)=0\right\} .
$$

Since both $\mathscr{P}_{1}$ and $\overline{\text { co }} \mathscr{P}_{1}$ are compact, it is immediate that $\mathscr{E} \overline{\text { co }} \mathscr{P}_{1} \subseteq \mathscr{E} \mathscr{P}_{1}$.

In fact the same proof yields the more general case for arbitrary unimodular $c$, i.e.,

$$
\mathscr{E} \overline{\mathrm{co}} \mathscr{P}_{c} \subseteq\left\{\left(\frac{1+c \varphi}{1-\varphi}\right)^{\beta}: \varphi \text { is an inner function with } \varphi(0)=0\right\} .
$$

Also

$$
\mathscr{E} \overline{\mathrm{co}}\left(\frac{\operatorname{St}(\alpha)}{z}\right)=\left\{\frac{1}{(1-y z)^{\varepsilon}}:|y|=1\right\} \quad(\text { see }[6]) .
$$

An application of Lemma 2 now yields the stated form.

In [5] it was shown that, for $k>2, \mathscr{V}_{k} \subseteq \mathscr{C}\left(\frac{1}{2} k-1\right)$ and that, for $k \geq 4$, $\overline{\operatorname{co}} \mathscr{V}_{k}=\overline{\operatorname{co}} \mathscr{C}\left(\frac{1}{2} k-1\right)$. These results can be extended to similar results relating $\mathscr{V}_{p, q}$ to $\mathscr{A}_{\alpha, \beta}$. 
Theorem 5. For $0 \leq p \leq q, \mathscr{V}_{p, q} \subseteq \mathscr{A}_{\alpha, \beta}$ where $\alpha=1-\frac{1}{2} p$ and $\beta=\frac{1}{2}(q-p)$.

Proof. Note that $p=q$ implies $\mathscr{V}_{p, q}=\mathscr{V}_{q, q}=\mathscr{A}_{\alpha, 0}$, where $q=2(1-\alpha)$. Thus, without loss of generality, suppose $0 \leq p<q$. We modify the argument given for $\mathscr{V}_{k}$ in $\left[17\right.$, p. 24]. Take any $f \in \mathscr{V}_{p, q}$. Then

$$
f^{\prime}(z)=\exp \left[-\int_{U} \log (1-x z) d \mu(x)\right], \quad \text { where } \int_{U} d \mu=p \text { and } \int_{U}|d \mu| \leq q .
$$

Since $\mathscr{V}_{p, q} \subseteq \mathscr{V}_{p, q^{\prime}}$ for $q<q^{\prime}$, it is enough to consider the case $\int|d \mu|=q$. Let

$$
\mu_{1}=\frac{1}{(p+q)}(|\mu|+\mu)
$$

and

$$
\mu_{2}=\frac{1}{(q-p)}(|\mu|-\mu)
$$

Then $\mu_{1}$ and $\mu_{2}$ are probability measures on $U$. Let

$$
s(z)=z \exp \left[-\int p \log (1-x z) d \mu_{1}(x)\right] .
$$

Then $s \in S t(\alpha)$, where $\alpha=1-\frac{1}{2} p$.

Now

$$
\frac{z f^{\prime}(z)}{s(z)}=\left\{\exp \left[-\int \log (1-x z)\left(d \mu_{1}-d \mu_{2}\right)\right]\right\}^{\beta} \quad \text { where } \beta=\frac{q-p}{2} .
$$

By ([17], Theorem 2.5) this function is subordinate to $((1+c z) /(1-z))^{\beta}$ for some $|c|=1$. By Lemma $1, f \in \mathscr{A}_{\alpha, \beta}$.

Corollary 6. For $0<p \leq q-2$,

$$
\overline{\mathrm{co}} \mathscr{V}_{p, q}^{\prime}=\overline{\mathrm{co}} \mathscr{A}_{\alpha, \beta}^{\prime}=\mathscr{F}_{\beta, \beta+\varepsilon},
$$

where

$$
\alpha=1-\frac{p}{2}, \quad \beta=\frac{q-p}{2} \quad \text { and } \quad \varepsilon=2(1-\alpha)=p .
$$

Proof. Each kernel $(1-x z)^{\beta} /(1-y z)^{\beta+\varepsilon}$ is in $\mathscr{V}_{p, q}^{\prime}$. Now $p \leq q-2$ yields $\beta \geq 1$. Hence, by Theorem 4 ,

$$
\mathscr{F}_{\beta, \beta+\varepsilon}=\overline{\operatorname{co}} \mathscr{A}_{\alpha, \beta}^{\prime} \subseteq \overline{\operatorname{co}} \mathscr{V}_{p, q}^{\prime} .
$$

By Theorem 5, $\overline{\operatorname{co}} \mathscr{V}_{p, q}^{\prime} \subseteq \overline{\mathrm{co}} \mathscr{A}_{\alpha, \beta}^{\prime}$.

Remark. The family $\overline{c o} \mathscr{V}_{p, q}^{\prime}$, for this range of values, was obtained in [3]. 


\section{Support points}

The following result for $\mathscr{A}_{\alpha, \beta}$ was obtained by the first author in [15]. For completeness we include the proof.

Theorem 7. Let $f_{0}$ be a support point of $\mathscr{A}_{\alpha, \beta}$.

i) If $\beta \geq 1$, then

$$
f_{0}^{\prime}(z)=\frac{(1-x z)^{\beta}}{(1-y z)^{\beta+\varepsilon}}, \quad \text { where }|x|=|y|=1 \quad \text { with } x \neq y .
$$

ii) If $0<\beta<1$, then

$$
f_{0}^{\prime}(z)=\left(\sum_{k=1}^{N} \lambda_{k} \frac{1+c x_{k} z}{1-x_{k} z}\right)^{\beta} \cdot \frac{1}{\left(1-y_{0} z\right)^{\varepsilon}}
$$

where $N$ is a positive integer, $0 \leq \lambda_{k} \leq 1, \sum_{k=1}^{N} \lambda_{k}=1,\left|x_{k}\right|=1$ for $k=1,2, \ldots, N,\left|y_{0}\right|=|c|=1$ and $c \neq-1$.

Remark. As pointed out in [15], if $\beta=1$ and $\alpha<1$, then the argument in [14] can be modified to show that each kernel $(1-x z) /(1-y z)^{1+\varepsilon},|x|=|y|=1$, $x \neq y$, is actually an exposed point of $\mathscr{A}_{\alpha, 1}^{\prime}$. Hence

$$
\Sigma \mathscr{A}_{\alpha, 1}^{\prime}=\left\{k^{\prime}(z ; x, y):|x|=|y|=1, x \neq y\right\}
$$

We suspect the same is true for $\mathscr{A}_{\alpha, \beta}$ when $\beta>1$, but this has yet to be demonstrated.

Proof of Theorem 7. i) Let $J$ be a continuous linear functional on $\mathscr{A}$ such that

$$
\operatorname{Re} J\left(f_{0}\right)=\max \left\{\operatorname{Re} J(f): f \in \mathscr{A}_{\alpha, \beta}\right\}
$$

and $\operatorname{Re} J$ is nonconstant on $\mathscr{A}_{\alpha, \beta}$. Define $L$ by $L\left(f^{\prime}\right)=J(f)$ for all $f$ in $\mathscr{A}_{0}=$ $\{f \in \mathscr{A}: f(0)=0\}$. Then $L$ extends to a continuous linear functional on $\mathscr{A}$, $\operatorname{Re} L$ peaks over $\mathscr{A}_{\alpha, \beta}^{\prime}$ at $f_{0}^{\prime}$ and $\operatorname{Re} L$ is nonconstant on $\mathscr{A}_{\alpha, \beta}^{\prime}$. For fixed $\beta$, let $\mathscr{P}_{c}$ denote the set of functions subordinate to the function $((1+c z) /(1-z))^{\beta}$. By Lemma 1 , we have $f_{0}^{\prime}(z)=p_{0}(z) \cdot s_{0}(z) / z$, where $s_{0} \in S t(\alpha)$ and $p_{0} \in \mathscr{P}_{c_{0}}$ for some $\left|c_{0}\right|=1$.

We first show that $c_{0} \neq-1$. If not, $p_{0}(z)=1$ for all $z$ in $D$ and $f_{0}^{\prime}(z)=$ $s_{0}(z) / z$. Since $1+\varepsilon x z^{n} \in \mathscr{P}_{1}$ for sufficiently small $\varepsilon(>0)$, for all $|x|=1$ and $n=1,2,3, \ldots$, we must have

$$
\operatorname{Re} L\left(\frac{s_{0}(z)}{z}\right) \geq \operatorname{Re} L\left(\left(1+\varepsilon x z^{n}\right) \cdot \frac{s_{0}(z)}{z}\right) .
$$


This implies that $\operatorname{Re} x L\left(z^{n-1} s_{0}(z)\right) \leq 0$ for all $|x|=1$ and hence $L\left(z^{n-1} s_{0}(z)\right)$ $=0$ for $n=1,2,3 \ldots$.

Since, for any $f$ in $\mathscr{A}_{\alpha, \beta}, z f^{\prime}(z) / s_{0}(z)=1+\sum_{n=1}^{\infty} a_{n} x^{n}$ is analytic in $D$, as in [13, p. 217], $L\left(f^{\prime}\right)=L\left(s_{0}(z) / z\right)$ and $L$ is constant on $\mathscr{A}_{\alpha, \beta}^{\prime}$. This contradiction shows that $c_{0} \neq-1$.

Next we show that $s_{0}$ is a support point of $S t(\alpha)$. To this end let

$$
\mathscr{F}=\left\{p_{0}(z) \cdot \frac{s(z)}{z}: s \in S t(\alpha)\right\}
$$

By Lemma $1 \mathscr{F}$ is a subset of $\mathscr{A}_{\alpha, \beta}^{\prime}$ and $f_{0}^{\prime}$ is in $\mathscr{F}$. Hence

$$
\operatorname{Re} L\left(f_{0}^{\prime}\right) \geq \operatorname{Re} L\left(p_{0}(z) \cdot \frac{s(z)}{z}\right)
$$

for all $s$ in $S t(\alpha)$. Define $L_{1}$ on $\mathscr{A}_{0}$ by $L_{1}(g)=L\left(p_{0}(z) \cdot g(z) / z\right)$. Then $L_{1}$ extends to a continuous linear functional on $\mathscr{A}$ and $\operatorname{Re} L_{1}$ peaks over $S t(\alpha)$ at $s_{0}$.

We claim that $\operatorname{Re} L_{1}$ is nonconstant on $\operatorname{St}(\alpha)$. If not, $\operatorname{Re} L\left(p_{o}(z) \cdot s(z) / z\right)$ is constant for all $s$ in $S t(\alpha)$. Let $s(z)=z /(1-x z)^{\varepsilon} \quad(|x|=1)$. We have $\operatorname{Re} L\left(p_{0}(z) /(1-x z)^{\varepsilon}\right)$ is constant for all $|x|=1$. Since $L\left(p_{0}(z) /(1-x z)^{\varepsilon}\right)$ is an analytic function on $|x| \leq 1$ and the Taylor coefficients of $1 /(1-z)^{\varepsilon}$ are all nonzero, $L\left(z^{n} \cdot p_{0}(z)\right)=0$ for $n=1,2,3, \ldots$ Again as in [13, p. 217], $L\left(f^{\prime}\right)=L\left(p_{0}\right)$ for all $f$ in $\mathscr{A}_{\alpha, \beta}$, a contradiction. Hence $s_{0}$ is a support point of $S t(\alpha)$. It is known ([9]) that $\Sigma S t(\alpha)=\left\{z /(1-x z)^{\varepsilon}:|x|=1\right\}$. Thus $s_{0}(z)=z /\left(1-y_{0} z\right)^{\varepsilon}$ for some $\left|y_{0}\right|=1$ and $f_{0}^{\prime}(z)=p_{0}(z) \cdot\left(1-y_{0} z\right)^{-\varepsilon}$ where $p_{0}$ is in $\mathscr{P}_{c_{0}}$ for some $c_{0} \neq-1$, $\left|c_{0}\right|=1$.

Now we show that $p_{0}$ is a support point of $\mathscr{P}_{c_{0}}$. Let $\mathscr{G}=\left\{p(z) \cdot\left(1-y_{0} z\right)^{-\varepsilon}\right.$ : $\left.p \in \mathscr{P}_{c_{0}}\right\}$. Again by Lemma $1, \mathscr{G}$ is a subset of $\mathscr{A}_{\alpha, \beta}^{\prime}$ and $f_{0}^{\prime}$ is in $\mathscr{G}$. Define $L_{2}$ on $\mathscr{A}$ by

$$
L_{2}(g)=L\left(g(z) \cdot\left(1-y_{0} z\right)^{-\varepsilon}\right) .
$$

Then $L_{2}$ is a continuous linear functional on $\mathscr{A}$ and $\operatorname{Re} L_{2}$ peaks over $\mathscr{P}_{c_{0}}$ at $p_{0}$. If $\operatorname{Re} L_{2}$ is constant over $\mathscr{P}_{c_{0}}$, then $\operatorname{Re} L\left(p(z) \cdot\left(1-y_{0} z\right)^{-\varepsilon}\right)$ is constant for all $p$ in $\mathscr{P}_{c_{0}}$. Since $\left(\left(1+c_{0} x z^{m}\right) /\left(1-x z^{m}\right)\right)^{\beta}$ is in $\mathscr{P}_{c_{0}}$ for all $|x|=1$ and $m=1,2,3, \ldots$, it is easy to see that $L\left(z^{m}\left(1-y_{0} z\right)^{-\varepsilon}\right)=0$ for $m=1,2,3, \ldots$. Once again, as in $\left[13\right.$, p. 217], $L\left(f^{\prime}\right)=L\left(\left(1-y_{0} z\right)^{-\varepsilon}\right)$ for all $f$ in $\mathscr{A}_{\alpha, \beta}$, a contradiction. Hence $p_{0}$ is a support point of $\mathscr{P}_{c_{0}}$.

Case (a): $\beta>1$. In this case it was shown in $[12$, p. 535$]$ that

$$
\Sigma \mathscr{P}_{c_{0}}=\left\{\left(\frac{1+c_{0} x z}{1-x z}\right)^{\beta}:|x|=1\right\} .
$$


Hence

$$
f_{0}^{\prime}(z)=\left(\frac{1+c_{0} x_{0} z}{1-x_{0} z}\right)^{\beta} \cdot \frac{1}{\left(1-y_{0} z\right)^{\varepsilon}}
$$

for some $\left|x_{0}\right|=1$. Let

$$
\mathscr{H}=\left\{h \in \mathscr{A}: h(z) \prec \frac{1}{(1-z)^{\beta+\varepsilon}}\right\}
$$

and

$$
\mathscr{K}=\left\{\left(1+c_{0} x_{0} z\right)^{\beta} h(z): h \in \mathscr{H}\right\} .
$$

As in $[17$, p. 19], univalence and convexity of $\log (1-z)$ yield

$$
h_{0}(z)=\frac{1}{\left(1-x_{0} z\right)^{\beta}} \cdot \frac{1}{\left(1-y_{0} z\right)^{\varepsilon}} \prec \frac{1}{(1-z)^{\beta+\varepsilon}} .
$$

Hence $f_{0}^{\prime} \in \mathscr{K}$. By now standard methods, it is easy to see that $\mathscr{K}$ is a compact subset of $\overline{c o} \mathscr{A}_{\alpha, \beta}^{\prime}$. Since $f_{0} \in \Sigma \mathscr{A}_{\alpha, \beta}$ implies that $f_{0} \in \Sigma \overline{\mathrm{co}} \mathscr{A}_{\alpha, \beta}$, Re $L$ peaks over $\mathscr{K}$ at $f_{0}^{\prime}$. By defining $L_{3}$ on $\mathscr{A}$ by $L_{3}(g)=L\left(\left(1+c_{0} x_{0} z\right)^{\beta} g(z)\right)$, as before one can show that $h_{0} \in \Sigma \mathscr{H}$. But

$$
\Sigma \mathscr{H}=\left\{\frac{1}{(1-x z)^{\beta+\varepsilon}}:|x|=1\right\} \quad[12, \text { p. 535]. }
$$

Hence $h_{0}(z)=1 /\left(1-u_{0} z\right)^{\beta+\varepsilon}$ for some $\left|u_{0}\right|=1$ and

$$
f_{0}^{\prime}(z)=\left(1+c_{0} x_{0} z\right)^{\beta} /\left(1-u_{0} z\right)^{\beta+\varepsilon} .
$$

This completes the proof of (i) when $\beta>1$.

Case (b): $\beta=1$. In this case it was shown in [12] that

$$
\begin{gathered}
\Sigma \mathscr{P}_{c_{0}}=\left\{\sum_{k=1}^{N} \lambda_{k} \frac{1+c_{0} x_{k} z}{1-x_{k} z}: 0 \leq \lambda_{k} \leq 1 \text { and }\left|x_{k}\right|=1 \text { for } k=1,2, \ldots, N,\right. \\
\text { and } \left.\sum_{k=1}^{N} \lambda_{k}=1 \text { for } N=1,2,3, \ldots\right\} .
\end{gathered}
$$

Hence $f_{0}^{\prime}(z)=\sum_{k=1}^{N} \lambda_{k}\left(1+c_{0} x_{k} z\right) /\left(1-x_{k} z\right) \cdot\left(1 /\left(1-y_{0} z\right)^{\varepsilon}\right)$.

The function $g_{k}$ defined by

$$
g_{k}^{\prime}(z)=\frac{1+c_{0} x_{k} z}{1-x_{k} z} \cdot \frac{1}{\left(1-y_{0} z\right)^{\varepsilon}}, \quad g_{k}(0)=0,
$$

is clearly in $\mathscr{A}_{\alpha, 1}$ for $k=1,2, \ldots, N$. Since $f_{0}(z)=\sum_{k=1}^{N} \lambda_{k} g_{k}(z)$ and $f_{0}$ is a support point of $\mathscr{A}_{\alpha, 1}$, each $g_{k}$ must be a support point of $\mathscr{A}_{\alpha, 1}$. An argument similar to that in case (a) (with $\beta=1$ ) implies that the denominator of each $g_{k}$ must collapse to a single factor of the form $\left(1-y_{k} z\right)^{1+\varepsilon}$. Hence $x_{k}=y_{0}$ for $k=1,2, \ldots, N$ and

$$
f_{0}^{\prime}(z)=\frac{1+c_{0} y_{0} z}{\left(1-y_{0} z\right)^{1+\varepsilon}}
$$

This completes the proof of (i). 
(ii) In this case, $0<\beta<1$, the function $\left(\left(1+c_{0} z\right) /(1-z)\right)^{\beta}$ is univalent and convex in $D$. It follows from Theorem 6 in $[12$, p. 531] that

$$
\Sigma \mathscr{P}_{c_{0}}=\left\{\left(\frac{1+c_{0} \varphi}{1-\varphi}\right)^{\beta}: \varphi \text { is a finite Blashke product with } \varphi(0)=0\right\} .
$$

Hence

$$
f_{0}^{\prime}(z)=\left(\frac{1+c_{0} \varphi_{0}}{1-\varphi_{0}}\right)^{\beta} \cdot \frac{1}{\left(1-y_{0} z\right)^{\varepsilon}}
$$

where $\varphi_{0}$ is a finite Blashke product with $\varphi_{0}(0)=0$. A trivial modification of the representation

$$
\frac{1+\varphi_{0}}{1-\varphi_{0}}=\sum_{k=1}^{N} \lambda_{k} \frac{1+x_{k} z}{1-x_{k} z}
$$

obtained in $[9$, p. 83$]$ yields

$$
\frac{1+c_{0} \varphi_{0}}{1-\varphi_{0}}=\sum_{k=1}^{N} \lambda_{k} \frac{1+c_{0} x_{k} z}{1-x_{k} z} .
$$

The form stated in (ii) for $f_{0}^{\prime}(z)$ follows.

Corollary 8. For $0 \leq p \leq q-2$, if $f$ is a support point of $\mathscr{V}_{p, q}$, then

$$
f^{\prime}(z)=\frac{\left(1-x_{0} z\right)^{(q-p) / 2}}{\left(1-y_{0} z\right)^{(q+p) / 2}}
$$

where $\left|x_{0}\right|=\left|y_{0}\right|=1$ and $x_{0} \neq y_{0}$. $(\geq 1)$.

Proof. By Theorem 5, $\mathscr{V}_{p, q} \subseteq \mathscr{A}_{\alpha, \beta}$, where $\alpha=1-\frac{1}{2} p$ and $\beta=\frac{1}{2}(q-p)$

By Corollary 6, $\overline{\text { co }} \mathscr{V}_{p, q}=\overline{\text { co }} \mathscr{A}_{\alpha, \beta}$. Hence each support point of $\mathscr{V}_{p, q}$ must be a support point of $\mathscr{A}_{\alpha, \beta}$. The Corollary then follows from Theorem 7 .

Next we obtain some information on support points of $\mathscr{V}_{p, q}$ for values of $p$ and $q$ which are not covered in Corollary 7 .

Theorem 2 in [8] shows that the representing measure for a support point of $\mathscr{V}_{p, q}^{\prime}$ must be discrete. That is, if $f$ is a support point of $\mathscr{V}_{p, q}$, then

$$
f^{\prime}(z)=\frac{\prod_{j=1}^{N}\left(1-x_{j} z\right)^{\alpha_{j}}}{\prod_{j=1}^{M}\left(1-y_{j} z\right)^{\beta_{j}}},
$$

where $N$ and $M$ are positive integers, $\alpha_{j}>0, \beta_{k}>0,\left|x_{j}\right|=\left|y_{k}\right|=1, x_{j} \neq y_{k}$, for $k=1, \ldots, M$ and $j=1,2, \ldots, N$ with

$$
\sum_{j=1}^{N} \alpha_{j}=\frac{q-p}{2} \quad \text { and } \quad \sum_{j=1}^{M} \beta_{j}=\frac{q+p}{2} .
$$

For $p \geq 0$, we will show that the denominator must collapse to one factor. 
Theorem 9. For $p \geq 0$ if $f$ is a support point of $\mathscr{V}_{p, q}$, then

$$
f^{\prime}(z)=\frac{\prod_{j=1}^{N}\left(1-x_{j} z\right)^{\alpha_{j}}}{\left(1-y_{0} z\right)^{(q+p) / 2}}
$$

where $\left|y_{0}\right|=1$, and $N, x_{j}$ and $\alpha_{j}$ are as above.

Proof. In [7] it was shown that if

$$
\mathscr{G}_{p}=\left\{\int_{U} \frac{1}{(1-x z)^{p}} d \mu(x): \mu \text { is a probability measure on } U\right\},
$$

then $\mathscr{G}_{p} \cdot \mathscr{G}_{q} \subseteq \mathscr{G}_{p+q}$ where $p>0$ and $q>0$. Hence

$$
\frac{1}{\prod_{j=1}^{M}\left(1-y_{j} z\right)^{\beta_{j}}} \in \mathscr{G}_{(q+p) / 2}
$$

and

$$
f^{\prime}(z)=\int \frac{G(z)}{(1-y z)^{(q+p) / 2}} d \mu(y), \quad \text { where } G(z)=\prod_{j=1}^{N}\left(1-x_{j} z\right)^{\alpha_{j}} .
$$

Note $G(z) /(1-y z)^{(q+p) / 2}$ is in $\mathscr{V}_{p, q}^{\prime}$ for all $|y|=1$. Using the fact that $f^{\prime}(z)$ is a support point of $\mathscr{V}_{p, q}^{\prime}$, by standard arguments one can show that $\mu$ is discrete.

Thus,

$$
f^{\prime}(z)=\sum_{k=1}^{L} \lambda_{k} \frac{G(z)}{\left(1-y_{k} z\right)^{(q+p) / 2}},
$$

where $L$ is a positive integer, $\lambda_{k} \geq 0,\left|y_{k}\right|=1$ for $k=1,2, \ldots, L$ and $\sum_{k=1}^{L} \lambda_{k}$ $=1$.

A comparison of the singularities of the two forms for $f^{\prime}(z)$ yields the result.

\section{Coefficient estimates}

Lemma 10. Let $a>0, b>0$ and $\left|c_{1}\right|=\left|c_{2}\right|=1$. If

$$
f(z) \prec\left(\frac{1+c_{1} z}{1-z}\right)^{a} \quad \text { and } \quad g(z) \prec\left(\frac{1+c_{2} z}{1-z}\right)^{b} \text {, }
$$

then there is a $c$ with $|c|=1$ such that $f(z) \cdot g(z) \prec((1+c z) /(1-z))^{a+b}$. 
Proof. Let $c_{j}=e^{2 i \theta_{j}}, j=1,2$. Then

$$
a\left(\theta_{1}-\pi / 2\right)<\arg f(z)<a\left(\theta_{1}+\pi / 2\right)
$$

and

$$
b\left(\theta_{2}-\pi / 2\right)<\arg g(z)<b\left(\theta_{2}+\pi / 2\right) .
$$

Hence

$$
\frac{\left(a \theta_{1}+b \theta_{2}\right)}{a+b}-\pi / 2<\arg (f(z) \cdot g(z))^{1 / a+b}<\frac{\left(a \theta_{1}+b \theta_{2}\right)}{a+b}+\pi / 2 .
$$

That is, $(f(z) \cdot g(z))^{1 / a+b} \prec(1+c z) /(1-z)$, where $c=e^{2 i\left(\left(a \theta_{1}+b \theta_{2}\right) /(a+b)\right)}$. This is the assertion in the Lemma.

A crucial ingredient in our proof of Theorem 13, which concerns the coefficient domination of $\mathscr{A}_{\alpha, \beta}$, is the following:

Lemma 11. (See [2], [4] and [17]). Let $\alpha \geq 1$. If $f \prec((1+c z) /(1-z))^{\alpha}$ for some $|c|=1$, then $f \ll((1+z) /(1-z))^{\alpha}$.

Note: If $f(z)=\sum_{n=0}^{\infty} a_{n} z^{n}$ and $g(z)=\sum_{n=0}^{\infty} b_{n} z^{n}$ we write $f \ll g$ to mean $\left|a_{n}\right| \leq\left|b_{n}\right|, n=1,2,3 \ldots$

We will also use the following elementary fact.

Lemma 12. Let $f_{1} \ll g_{1}$ and $f_{2} \ll g_{2}$. If both $g_{1}$ and $g_{2}$ have nonnegative coefficients in their power series expansions, then $f_{1} f_{2} \ll g_{1} g_{2}$.

Theorem 13. Let $\beta+\frac{1}{2} \varepsilon \geq 1$. If $f \in \mathscr{A}_{\alpha, \beta}$ with $\varepsilon=2(1-\alpha)$, then $f^{\prime}(z) \ll(1+z)^{\beta} /(1-z)^{\beta+\varepsilon}$.

Proof. The argument employs the same device introduced in [5]. By Lemma 1 we have, for any $f \in \mathscr{A}_{\alpha, \beta}$,

$$
f^{\prime}(z)=[p(z)]^{\beta} \frac{s(z)}{z} \quad \text { where } \quad p(z) \prec \frac{1+c z}{1-z}, \quad|c|=1 \text { and } s \in \operatorname{St}(\alpha) .
$$

As noted earlier

$$
s(z)=\int \frac{z}{(1-x z)^{\varepsilon}} d \mu(x),
$$

where $\mu$ is a probability measure on $U$. It clearly suffices to prove the result when $\mu$ is a unit point mass. Thus, without loss of generality, suppose,

$$
f^{\prime}(z)=[p(z)]^{\beta} \frac{1}{\left(1-x_{0} z\right)^{\varepsilon}}=[p(z)]^{\beta}\left(\frac{1+x_{0} z}{1-x_{0} z}\right)^{\varepsilon / 2} \frac{1}{\left(1-x_{0}^{2} z^{2}\right)^{\varepsilon / 2}} .
$$


By Lemma 10

$$
[p(z)]^{\beta}\left(\frac{1+x_{0} z}{1-x_{0} z}\right)^{\varepsilon / 2} \prec\left(\frac{1+c_{0} z}{1-z}\right)^{\beta+\varepsilon / 2}
$$

with $\left|c_{0}\right|=1$. Since $\beta+\frac{1}{2} \varepsilon \geq 1$, by Lemma 11

$$
[p(z)]^{\beta}\left(\frac{1+x_{0} z}{1-x_{0} z}\right)^{\varepsilon / 2} \ll\left(\frac{1+z}{1-z}\right)^{\beta+\varepsilon / 2}=g_{1}(z) .
$$

Also

$$
\frac{1}{\left(1-x_{0}^{2} z^{2}\right)^{\varepsilon / 2}} \ll \frac{1}{\left(1-z^{2}\right)^{\varepsilon / 2}}=g_{2}(z) .
$$

But both $g_{1}(z)$ and $g_{2}(z)$ have nonnegative coefficients. Hence, by Lemma 12,

$$
f^{\prime}(z) \ll\left(\frac{1+z}{1-z}\right)^{\beta+\varepsilon / 2} \frac{1}{\left(1-z^{2}\right)^{\varepsilon / 2}}=\frac{(1+z)^{\beta}}{(1-z)^{\beta+\varepsilon / 2}} .
$$

As corollaries we easily obtain the following results which appear in [3].

Corollary 14. Let $p \geq 0$ and $q \geq \max \{p, 2\}$. If $f \in \mathscr{V}_{p, q}$ then

$$
f^{\prime}(z) \ll \frac{(1+z)^{(q-p) / 2}}{(1-z)^{(q+p) / 2}} .
$$

Proof. Since $\mathscr{V}_{p, q} \subseteq \mathscr{A}_{\alpha, \beta}$ for the stated range of parameters, the proof is immediate from the theorem.

Remark. In [3] the corollary above was obtained by introducing the family $S_{\alpha, \beta}$. A function $f$ in $\mathscr{A}$ belongs to $S_{\alpha, \beta}$ if $f(z)=h_{1}(z) / h_{2}(z)$ where $h_{1} \in S t(\alpha)$ and $h_{2} \in S t(\beta)$. It was shown there that $S_{\alpha, \beta}=\mathscr{V}_{p, q}^{\prime}$, where $\alpha=1-\frac{1}{4}(q+p)$ and $\beta=1-\frac{1}{4}(q-p)$. Hence Corollary 14 above is equivalent to the following result in [3].

Corollary 15. Let $\alpha \leq \beta<1$ and $\alpha+\beta<1$. If $f \in S_{\alpha, \beta}$ then

$$
f(z) \ll \frac{(1+z)^{2(1-\beta)}}{(1-z)^{2(1-\alpha)}} .
$$

We turn now to the case $\beta+\frac{1}{2} \varepsilon<1$.

Theorem 16. Let $\beta>0, \varepsilon>0$ and $\beta+\frac{1}{2} \varepsilon<1$. Then there is a function $f \in \mathscr{V}_{p, q}$ (hence also in $\mathscr{A}_{\alpha, \beta}$ ) such that the second coefficient in the power series for $f^{\prime}(z)$ has modulus strictly greater than the second coefficient in the power series for $(1+z)^{\beta} /(1-z)^{\beta+\varepsilon}$. (That is, the condition $\beta+\frac{1}{2} \varepsilon \geq 1$ is sharp in Theorem 13.) 
Before we prove the theorem let us introduce some notation and present some preliminary calculations. Using Lemma 1 , it is an easy calculation to show that, for any $f \in \mathscr{A}_{\alpha, \beta}$, if $f^{\prime}(z)=1+\sum_{n=1}^{\infty} a_{n} z^{n}$, then $\left|a_{1}\right| \leq 2 \beta+\varepsilon=b_{1}$, where

$$
\frac{(1+z)^{\beta}}{(1-z)^{\beta+\varepsilon}}=1+\sum_{n=1}^{\infty} b_{n} z^{n}
$$

The proof of Theorem 16 arose as a result of the investigation of the second coefficient.

Write

$$
\frac{(1+x z)^{\beta / 2}(1+y z)^{\beta / 2}}{(1-z)^{\beta+\varepsilon}}=1+\sum_{n=1}^{\infty} a_{n}(x, y) z^{n},
$$

where $|x|=|y|=1$. Let

$$
\begin{gathered}
A=(\beta+\varepsilon)(\beta+\varepsilon+1), \\
B=2 \beta(\beta+\varepsilon),
\end{gathered}
$$

and

$$
C=\beta-\beta^{2} \text {. }
$$

Then

$$
2 a_{2}(x, y)=A+B\left(\frac{x+y}{2}\right)+\beta^{2}\left(\frac{x+y}{2}\right)^{2}-\beta\left(\frac{x^{2}+y^{2}}{2}\right)
$$

and

$$
2 a_{2}(x, x)=A+B x-C x^{2} .
$$

Lemma 17. Let $\beta>0, \varepsilon \geq 0$ and $\beta+\frac{1}{2} \varepsilon<1$.

i) $\max \left\{\left|a_{2}(x, x)\right|:|x|=1\right\}=a_{2}(1,1)$ if and only if $\sqrt{2+\varepsilon} \leq 2 \beta+\varepsilon<2$.

ii) If $2 \beta+\varepsilon<\sqrt{2+\varepsilon}$, then $\max \left\{\left|a_{2}(x, x)\right|:|x|=1\right\}$ occurs at $x=x_{0}$, where $\operatorname{Re} x_{0}=B(A-C) / 4 A C$.

Proof. Since $\left|2 a_{2}(x, x)\right|^{2}=A^{2}+B^{2}+C^{2}+2 A B \operatorname{Re} x-2 B C \operatorname{Re} x-2 A C \operatorname{Re} x^{2}$, if we let $t=\operatorname{Re} x$, then the right-hand side of this equation equals

$$
G(t)=(A+C)^{2}+B^{2}+2 B(A-C) t-4 A C t^{2} .
$$

If $0<\beta<1$, then $A C>0$. Thus $\max _{|t|<1} g(t)$ occurs at $t_{0}=B(A-C) / 4 A C>0$ provided $t_{0}<1$, i.e. provided $B(A-C)<4 A C$. If $t_{0} \geq 1$, then $\max _{|t| \leq 1} g(t)$ occurs at $t_{0}=1$. A simple calculation shows that the inequality $B(A-C)<4 A C$ is equivalent to $2 \beta+\varepsilon<\sqrt{2+\varepsilon}$ and the lemma follows. 
Remark. For some special values of $\beta$ and $\varepsilon$ this lemma appeared in [4]. For later use we wish to note that, in the case $2 \beta+\varepsilon<\sqrt{2+\varepsilon}$, the actual value of the maximum of $\left|a_{2}(x, x)\right|^{2}$ is given by

$$
\max _{|x|=1}\left|a_{2}(x, x)\right|^{2}=\left|a_{2}\left(x_{0}, x_{0}\right)\right|^{2}=\frac{(A+C)^{2}\left(4 A C+B^{2}\right)}{4 A C} .
$$

Proof of the theorem. Our goal is to construct $f \in \mathscr{V}_{p, q}$ with the property that, if $f^{\prime}(z)=\sum_{n=0}^{\infty} a_{n} z^{n}$ and $(1+z)^{\beta} /(1-z)^{\beta+\varepsilon}=\sum_{n=0}^{\infty} b_{n} z^{n}$, then $\left|a_{2}\right|>\left|b_{2}\right|$. To this end let $f \in \mathscr{V}_{p, q}$, where

$$
f^{\prime}(z)=\frac{(1+x z)^{\beta / 2}(1+\bar{x} z)^{\beta / 2}}{(1-z)^{\beta+\varepsilon}}=1+\sum_{n=1}^{\infty} a_{n}(x, \bar{x}) z^{n}
$$

in our notation above.

Write $h(t)=2 a_{2}(x, \bar{x})=A+\beta+B t-(C+\beta) t^{2}$ where $t=\operatorname{Re} x$ and note that $h(t)$ is real. An easy calculation shows that $h(t)$ peaks at $t_{1}=B / 2(C+\beta)$ and $t_{1}<1$ is equivalent to $\beta+\frac{1}{2} \varepsilon<1$.

Case (i): $\sqrt{2+\varepsilon} \leq 2 \beta+\varepsilon<2$. In this case, by Lemma 17 and the above calculation, we have

$$
\max _{|t| \leq 1} h^{2}(t)=h^{2}\left(t_{1}\right)>h^{2}(1)=g(1)=\max _{|t| \leq 1} g(t) .
$$

Consequently $\left|a_{2}\left(x_{1}, \bar{x}_{1}\right)\right|>\left|a_{2}(1,1)\right|$, where $\operatorname{Re} x_{1}=t_{1},\left|x_{1}\right|=1$.

Case (ii): $2 \beta+\varepsilon<\sqrt{2+\varepsilon}$. In this case $g\left(t_{0}\right)=\max _{|t| \leq 1} g(t)$ (Lemma 17 (ii)).

We claim that

$$
h^{2}\left(t_{1}\right)=\max _{|t| \leq 1} h^{2}(t)>g\left(t_{0}\right)=\max _{|t| \leq 1} g(t) .
$$

A simple computation shows that

$$
h\left(t_{1}\right)=A+\beta+\frac{B^{2}}{4(\beta+C)} .
$$

We observed earlier that

$$
g\left(t_{0}\right)=\frac{(A+C)^{2}\left(B^{2}+4 A C\right)}{4 A C} .
$$

Hence to prove the claim, for

$$
0<\beta \leq \frac{1}{2}(\sqrt{\varepsilon+2}-\varepsilon),
$$


we show that $h^{2}\left(t_{1}\right)>g\left(t_{0}\right)$, i.e.

$$
4(A+C)^{2}\left(B^{2}+4 A C\right)(\beta+C)^{2}-A C\left[4(A+\beta)(\beta+C)+B^{2}\right]^{2}<0 .
$$

In the following, equivalent forms of the inequalities are obtained after dividing out by common factors. Since

$$
\begin{aligned}
A+C & =(2 \beta+\varepsilon)(\varepsilon+1), \\
B^{2}+4 A C & =4 \beta(\beta+\varepsilon)(\varepsilon+1),
\end{aligned}
$$

and

$$
4(A+\beta)(C+\beta)+B^{2}=4 \beta[2(2 \beta+\varepsilon)(\varepsilon+1)-\beta \varepsilon],
$$

the inequality $(*)$ above is equivalent to

$$
(2 \beta+\varepsilon)^{2}(1+\varepsilon)^{3}(2-\beta)^{2}-(\beta+\varepsilon+1)(1-\beta)[2(2 \beta+\varepsilon)(\varepsilon+1)-\beta \varepsilon]^{2}<0 .
$$

To simplify this inequality, write $u=2 \beta+\varepsilon$ and $v=1+\varepsilon$. Since

$$
(\beta+\varepsilon+1)(1-\beta)=(\varepsilon+1)-\beta(\varepsilon+1)+\beta(1-\beta),
$$

the inequality $(*)$ above is equivalent to

$$
u^{2} v^{3}\left(4-4 \beta+\beta^{2}\right)-[v-\beta v+\beta(1-\beta)] \cdot\left[4 u^{2} v^{2}-4 \beta \varepsilon u v+\beta^{2} \varepsilon^{2}\right]<0,
$$

which is the same as

$$
\begin{aligned}
4 u^{2} v^{3} & -4 \beta u^{2} v^{3}+\beta^{2} u^{2} v^{3}-4 u^{2} v^{3}+4 \beta \varepsilon u v^{2}-\beta^{2} \varepsilon^{2} v+4 \beta u^{2} v^{3} \\
& -4 \beta^{2} \varepsilon u v^{2}+\beta^{3} \varepsilon^{2} v-4 \beta(1-\beta) u^{2} v^{2}+4 \beta^{2}(1-\beta) \varepsilon u v-\beta^{3}(1-\beta) \varepsilon^{2}<0 .
\end{aligned}
$$

This is equivalent to

$$
\begin{aligned}
\beta u^{2} v^{3} & +4 \varepsilon u v^{2}-\beta \varepsilon^{2} v-4 \beta \varepsilon u v^{2}+\beta^{2} \varepsilon^{2} v-4(1-\beta) u^{2} v^{2} \\
& +4 \beta(1-\beta) \varepsilon u v-\beta^{2}(1-\beta) \varepsilon^{2}<0 .
\end{aligned}
$$

Now we put $u=2 \beta+\varepsilon$ back in and obtain the equivalent inequality

$$
\begin{aligned}
\left(4 v^{3}\right. & \left.+16 v^{2}-8 \varepsilon v+\varepsilon^{2}\right) \beta^{2}+\left(4 \varepsilon v^{3}+8 \varepsilon v^{2}+\varepsilon^{2} v-16 v^{2}+8 \varepsilon v\right) \beta \\
& +\varepsilon^{2} v^{3}-8 \varepsilon v^{2}+3 \varepsilon^{2} v<0 .
\end{aligned}
$$

If we put $v=\varepsilon+1$ back in, the inequality has the form

$$
R \beta^{2}+S \beta+T>0
$$


where

$$
\begin{aligned}
R & =-4 \varepsilon^{3}-21 \varepsilon^{2}-36 \varepsilon-20=-(\varepsilon+2)^{2}(4 \varepsilon+5), \\
S & =-4 \varepsilon^{4}-17 \varepsilon^{3}-16 \varepsilon^{2}+12 \varepsilon+16=-(\varepsilon+2)^{2}\left(4 \varepsilon^{2}+\varepsilon-4\right)
\end{aligned}
$$

and

$$
T=-(\varepsilon+1)\left(\varepsilon^{4}+2 \varepsilon^{3}-4 \varepsilon^{2}-8 \varepsilon\right)=-\varepsilon(\varepsilon+1)(\varepsilon-2)(\varepsilon+2)^{2} .
$$

This inequality is clearly equivalent to

$$
F_{\varepsilon}(\beta)=(4 \varepsilon+5) \beta^{2}+\left(4 \varepsilon^{2}-\varepsilon-4\right) \beta-\varepsilon(2-\varepsilon)(\varepsilon+1)<0 .
$$

To verify $(*)$ for $0<\beta<\frac{1}{2}(\sqrt{\varepsilon+2}-\varepsilon)$, it is sufficient to check the last inequality at the end points $\beta_{0}=0$ and $\beta_{1}=\frac{1}{2}(\sqrt{\varepsilon+2}-\varepsilon)$. Since $\varepsilon<2$, clearly $F_{\varepsilon}(0)<0$.

A straightforward calculation gives

$$
F_{\varepsilon}\left(\beta_{1}\right)=\frac{1}{4}(1+\varepsilon)(10+3 \varepsilon-8 \sqrt{2+\varepsilon}) .
$$

So $F_{\varepsilon}\left(\beta_{1}\right)<0 \Leftrightarrow(10+3 \varepsilon)^{2}-64(2+\varepsilon)<0$.

This is the same as $(\varepsilon-2)(9 \varepsilon+14)<0$. Since $\varepsilon<2$, we have $F_{\varepsilon}(\beta)<0$ for all $0<\beta<\frac{1}{2}(\sqrt{\varepsilon+2}-\varepsilon)$. Hence we have completed the proof of our claim.

\section{Closed convex hulls when $\beta+\frac{1}{2} \varepsilon<1$}

From the proof of Theorem 15 it follows that, for $\beta+\frac{1}{2} \varepsilon<1$, there is an $x_{0}$ with $\left|x_{0}\right|=1$ such that, if

$$
f_{0}^{\prime}(z)=\frac{\left(1+x_{0} z\right)^{\beta / 2}\left(1+\bar{x}_{0} z\right)^{\beta / 2}}{(1-z)^{\beta+\varepsilon}}=1+\sum_{n=1}^{\infty} a_{n}\left(x_{0}, \bar{x}_{0}\right) z^{n}
$$

then $\left|a_{2}\left(x_{0}, \bar{x}_{0}\right)\right|>\max _{|x|=1}\left|a_{2}(x, x)\right|$. We claim this also shows that

$$
\overline{\operatorname{co}} \mathscr{V}_{p, q}^{\prime} \supsetneqq \mathscr{F}_{\beta, \beta+\varepsilon}, \quad \text { where } \varepsilon=p \text { and } \beta=\frac{1}{2}(q-p) .
$$

Since $k(z ; x, y) \in \mathscr{V}_{p, q}$ for $|x|=|y|=1$, we know $\mathscr{F}_{\beta, \beta+\varepsilon} \subseteq \overline{\text { co }} \mathscr{V}_{p, q}^{\prime}$. However, it follows from Lemmas 11 and 12 that $k^{\prime}(z ; x, y) \ll(1+z)^{\beta} /(1-z)^{\beta+\varepsilon}$ for all $|x|=|y|=1$. Since $\mathscr{F}_{\beta, \beta+\varepsilon}=\overline{\mathrm{co}}\left\{k^{\prime}(z ; x, y):|x|=|y|=1\right\}$, we also have

$$
\mathscr{F}_{\beta, \beta+\varepsilon} \ll \frac{(1+z)^{\beta}}{(1-z)^{\beta+\varepsilon}}=1+\sum_{n=1}^{\infty} b_{n} z^{n} .
$$

The lack of domination of $\mathscr{V}_{p, q}^{\prime}$ by $b_{2}$, however, shows that $\overline{\mathrm{co}} \mathscr{V}_{p, q}^{\prime}$ is strictly larger than $\mathscr{F}_{\beta, \beta+\varepsilon}$. We can say even more. 
Theorem 18. For $\beta+\frac{1}{2} \varepsilon<1$,

$$
\overline{\operatorname{co}} \mathscr{A}_{\alpha, \beta}^{\prime} \supsetneqq \overline{\operatorname{co}} \mathscr{V}_{\varepsilon, 2 \beta+\varepsilon}^{\prime} \supsetneqq \mathscr{F}_{\beta, \beta+\varepsilon},
$$

where, as always, $\varepsilon=2(1-\alpha)$.

Proof. It remains to show that $\overline{\operatorname{co}} \mathscr{V}_{\varepsilon, 2 \beta+\varepsilon}^{\prime} \neq \overline{\operatorname{co}} \mathscr{A}_{\alpha, \beta}^{\prime}$ (equivalently $\overline{\operatorname{co}} \mathscr{V}_{\varepsilon, 2 \beta+\varepsilon}$ $\left.\neq \overline{\mathrm{co}} \mathscr{A}_{\alpha, \beta}\right)$. If $\overline{\mathrm{co}} \mathscr{V}_{\varepsilon, 2 \beta+\varepsilon}=\overline{\mathrm{co}} \mathscr{A}_{\alpha, \beta}$, then each support point of $\mathscr{V}_{\varepsilon, 2 \beta+\varepsilon}$ is also a support point of $\mathscr{A}_{\alpha, \beta}$. This follows from the general fact that, if $\mathscr{F}$ and $\mathscr{G}$ are compact subsets of $\mathscr{A}$ such that $\mathscr{F} \subseteq \mathscr{G}$ and $\overline{\mathrm{co}} \mathscr{F}=\overline{\mathrm{co}} \mathscr{G}$, then each support point of $\mathscr{F}$ is also a support point of $\mathscr{G}$. Take any $f \in \Sigma \mathscr{V}_{\varepsilon, 2 \beta+\varepsilon} \subseteq \Sigma \mathscr{A}_{\alpha, \beta}$. Then Theorem 9 implies that

$$
f^{\prime}(z)=\frac{\prod_{j=1}^{N}\left(1-x_{j} z\right)^{\alpha_{j}}}{\left(1-y_{0} z\right)^{\beta+\varepsilon}},
$$

and, since $\beta<1$, Theorem 7 implies that

$$
f^{\prime}(z)=\left(\sum_{k=1}^{N} \lambda_{k} \frac{1+c x_{k} z}{1-x_{k} z}\right)^{\beta} \cdot \frac{1}{\left(1-x_{0} z\right)^{\varepsilon}} .
$$

Comparison of the singularities of the two forms for $f^{\prime}(z)$ yields $x_{k}=x_{0}=y_{0}$ for all $k=1,2, \ldots, N$. Hence

$$
f^{\prime}(z)=\frac{\left(1-x_{0} z\right)^{\beta}}{\left(1-y_{0} z\right)^{\beta+\varepsilon}}
$$

Now we have

$$
\Sigma \mathscr{V}_{\varepsilon, 2 \beta+\varepsilon}^{\prime} \subseteq\left\{\frac{(1-x z)^{\beta}}{(1-y z)^{\beta+\varepsilon}}:|x|=|y|=1\right\}
$$

from which we deduce $\overline{\mathrm{co}} \mathscr{V}_{\varepsilon, 2 \beta+\varepsilon}^{\prime}=\mathscr{F}_{\beta, \beta+\varepsilon}$. This contradiction when $\beta+\frac{1}{2} \varepsilon<1$ completes the proof.

Thus, for $\beta \geq 1$, we have $\overline{\operatorname{co}} \mathscr{V}_{\varepsilon, 2 \beta+\varepsilon}^{\prime}=\overline{\operatorname{co}} \mathscr{A}_{\alpha, \beta}^{\prime}=\mathscr{F}_{\beta, \beta+\varepsilon}$. For $\beta+\frac{1}{2} \varepsilon<1$ they are all distinct. The most intriguing and seemingly difficult open question involves the range $0<\beta<1$ and $\beta+\frac{1}{2} \varepsilon \geq 1$. In this range there is coefficient domination, i.e.

$$
\mathscr{V}_{\varepsilon, 2 \beta+\varepsilon}^{\prime} \ll \mathscr{A}_{\alpha, \beta}^{\prime} \ll \mathscr{F}_{\beta, \beta+\varepsilon} \ll \frac{(1+z)^{\beta}}{(1-z)^{\beta+\varepsilon}}
$$

but the question of equality of the closed convex hulls remains open. In particular, the longstanding question of identifying $\mathscr{E} \overline{\mathrm{co}} \mathscr{C}(\beta)=\mathscr{E} \overline{\mathrm{co}} \mathscr{A}_{0, \beta}, 0<\beta<1$, still remains inaccessible. 


\section{References}

[1] Abu-Muhana, Y., and T.H. MacGregor: Extreme poirts of functions subordinate to convex mappings. - Math. Z. 176, 1981, 511-519.

[2] Aharonov, D., and S. Friedland: On an inequality connected with the coefficient conjecture for functions of bounded boundary rotation. - Ann. Acad. Sci. Fenn. A I Math. 524, 1972.

[3] Aharonov, D., and S. Friedland: On functions of bounded boundary rotation. - Ann. Acad. Sci Fenn. A I Math. 585, 1974, 1-18.

[4] Brannan, D.A.: On coefficient problems for certain power series. - London Math. Soc. Lecture Note Ser. 12, 1973, 17-27.

[5] Brannan, D.A., J.G. Clunie, and W.E. Kirwan: On the coefficient problem for functions of bounded boundary rotation. - Ann. Acad. Sci. Fenn. Ser. A I Math. 523 1973, 1-18.

[6] Brickman, L., D.J. Hallenbeck, T.H. MacGregor, and D.R. Wilken: Convex hulls and extreme points of families of starlike and convex mappings. - Trans. Amer. Math. Soc. 185, 1973, 413-428.

[7] Brickman, L., T.H. MacGregor, and D.R. Wilken: Convex hulls of some classical families of univalent functions. - Trans. Amer. Math. Soc. 156, 1971, 911-107.

[8] Brickman, L., and D.R. Wilken: Measures and extremal problems. - J. Indian Math. Soc. $49,1985,127-149$.

[9] Cochrane, P.C., and T.H. MacGregor: Frechet differentiable functionals and support points for families of analytic functions. - Trans. Amer. Math. Soc. 236, 1978, 75-92.

[10] Goodman, A.W.: On close-to-convex functions of higher order. - Ann. Univ. Sci. Budapest 15, 1972, 17-30.

[11] Grassmann, E., W. Hengartner, and G. Schober: Support points of the class of close-to-convex functions. - Canadian Math. Bull. 19:2, 1976, 177-179.

[12] Hallenbeck, D.J., and T.H. MacGregor: Support points of families of analytic functions described by subordination. - Trans. Amer. Math. Soc. 278, 1983, 523-546.

[13] Hengartner, W., A. Pfluger, and G. Schober: On support points in the class of functions with bounded boundary rotation. - Ann. Acad. Sci. Fenn. Ser. A I Math. 6, 1981, 213-224.

[14] Hornblower, R., and D.R. WiLKEN: On the support points of close-to-convex functions. - Houston J. Math. 10:4, 1984, 593-599.

[15] Perera, S.: Support points and extreme points of some classes of analytic functions. Ph.D. dissertation, SUNY at Albany, 1983.

[16] Pommerenke, Сh.: On close-to-convex analytic functions. - Trans. Amer. Math. Soc. 144, 1965, 176-186.

[17] Schober, G.: Univalent functions-Selected topics. - Springer-Verlag, Berlin, 1975.

Received 7 November 1990

Revised 27 January 1992 